A method to stabilise the performance of negatively fed KM3NeT photomultipliers

This content has been downloaded from IOPscience. Please scroll down to see the full text.

2016 JINST 11 P12014

(http://iopscience.iop.org/1748-0221/11/12/P12014)

View the table of contents for this issue, or go to the journal homepage for more

Download details:

IP Address: 147.83.45.26

This content was downloaded on 07/03/2017 at 16:12

Please note that terms and conditions apply.

You may also be interested in:

The KM3NeT neutrino telescope

$\mathrm{R}$ Coniglione and $\mathrm{KM} 3 \mathrm{NeT}$ collaboration

KM3NeT: designing a cubic-kilometre scale neutrino telescope for the mediterranean

J Perkin and L F Thompson

Solar neutrino problem still perplexes

Dave Wark

KM3NeT - ORCA: measuring the neutrino mass ordering in the Mediterranean

Antoine Kouchner

High energy neutrino detection with KM3NeT

Pasquale Migliozzi and KM3NeT Collaboration

Photon detection efficiency measurements of the VERITAS Cherenkov telescope photomultipliers after four years of operation

E. Gazda, T. Nguyen, N. Otte et al.

Status of the KM3NeT project

A Margiotta

Very low power, high voltage base for a Photo Multiplier Tube for the KM3NeT deep sea neutrino

telescope

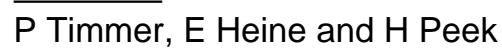

Progress and latest results from Baikal, Nestor, NEMO and KM3NeT

Emilio Migneco 


\section{A method to stabilise the performance of negatively fed KM3NeT photomultipliers}

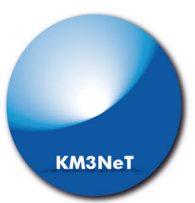

\section{The K3MNet collaboration}

S. Adrián-Martínez, ${ }^{a m}$ M. Ageron, ${ }^{b}$ S. Aiello, ${ }^{s}$ A. Albert, ${ }^{a n}$ F. Ameli, ${ }^{w}$ E. G. Anassontzis, ${ }^{a d}$ M. Andre, ${ }^{a l}$ G. Androulakis, ${ }^{a b}$ M. Anghinolfi, ${ }^{t}$ G. Anton, ${ }^{g}$ M. Ardid, ${ }^{a m}$ T. Avgitas, ${ }^{c}$ G. Barbarino, ${ }^{u, a i}$ E. Barbarito, ${ }^{q}$ B. Baret, ${ }^{c}$ J. Barrios-Martí, ${ }^{k}$ A. Belias, ${ }^{a b}$ E. Berbee, ${ }^{f}$ A. van den Berg, ${ }^{y}$ V. Bertin, ${ }^{b}$ S. Beurthey, ${ }^{b}$ V. van Beveren, ${ }^{f}$ N. Beverini, ${ }^{a j, v}$ S. Biagi, ${ }^{o}$ A. Biagioni, ${ }^{w}$ M. Billault, ${ }^{b}$ M. Bondì, ${ }^{s}$ R. Bormuth, ${ }^{f, z}$ B. Bouhadef, ${ }^{v}$ G. Bourlis, ${ }^{j}$ S. Bourret, ${ }^{c}$ C. Boutonnet, ${ }^{c}$ M. Bouwhuis, ${ }^{f}$ C. Bozza, ${ }^{a t}$ R. Bruijn, ${ }^{a q}$ J. Brunner, ${ }^{b}$ E. Buis, ${ }^{a g}$ R. Buompane, ${ }^{u, a e}$ J. Busto, ${ }^{b}$ G. Cacopardo, ${ }^{o}$ L. Caillat, ${ }^{b}$ M. Calamai, ${ }^{v}$ D. Calvo, ${ }^{k}$ A. Capone, ${ }^{a k, w}$ L. Caramete,$^{x}$ S. Cecchini, ${ }^{r}$ S. Celli, ${ }^{a k, w, i}$ C. Champion, ${ }^{c}$ S. Cherubini, ${ }^{o, a r}$ V. Chiarella, ${ }^{p}$ L. Chiarelli, ${ }^{r, n}$ T. Chiarusi, ${ }^{r}$ M. Circella, ${ }^{q}$ L. Classen, ${ }^{g}$ D. Cobas, ${ }^{c}$ R. Cocimano, ${ }^{o}$ J.A.B. Coelho, ${ }^{c}$ A. Coleiro, ${ }^{c}$ S. Colonges, ${ }^{c}$ R. Coniglione, ${ }^{o}$ M. Cordelli, ${ }^{p}$ A. Cosquer, ${ }^{b}$ P. Coyle, ${ }^{b}$ A. Creusot, ${ }^{c}$ G. Cuttone, ${ }^{o}$ C. D'Amato, ${ }^{o}$ A. D'Amico, ${ }^{f}$ A. D’Onofrio, ${ }^{u, a e}$ G. De Bonis, ${ }^{w}$ C. De Sio, ${ }^{a t}$ F. Di Capua, ${ }^{u}$ I. Di Palma, ${ }^{a k, w}$ C. Distefano, ${ }^{o}$ C. Donzaud, ${ }^{c}$ D. Dornic, ${ }^{b}$ Q. Dorosti-Hasankiadeh, ${ }^{y}$ E. Drakopoulou, ${ }^{a b}$ D. Drouhin, ${ }^{a n}$ M. Durocher, ${ }^{o, i}$ T. Eberl, ${ }^{g}$ S. Eichie, ${ }^{g, h}$ D. van Eijk, ${ }^{f}$ I. El Bojaddaini, ${ }^{a o}$ D. Elsaesser, ${ }^{a u}$ A. Enzenhöfer, ${ }^{b}$ M. Favaro, ${ }^{r, n}$ P. Fermani, ${ }^{w}$ G. Ferrara, ${ }^{o, a r}$ G. Frascadore, ${ }^{o}$ M. Furini, ${ }^{r}$ L.A. Fusco, ${ }^{r, a h}$ T. Gal, ${ }^{g}$ S. Galatà, ${ }^{c}$ F. Garufi, ${ }^{u, a i}$ P. Gay, ${ }^{c, m}$ M. Gebyehu, ${ }^{f}$ F. Giacomini, ${ }^{r, n}$ L. Gialanella, ${ }^{u, a e}$ V. Giordano, ${ }^{s}$ N. Gizani, ${ }^{j}$ R. Gracia, ${ }^{c}$ K. Graf, ${ }^{g}$ T. Grégoire, ${ }^{c}$ G. Grella, ${ }^{a t}$ A. Grmek, ${ }^{o}$ M. Guerzoni, ${ }^{r}$ R. Habel, ${ }^{p}$ S. Hallmann, ${ }^{g}$ H. van Haren, ${ }^{a c}$ S. Harissopulos, ${ }^{a b}$ T. Heid, ${ }^{g}$ A. Heijboer, ${ }^{f}$ E. Heine,${ }^{f}$ S. Henry, ${ }^{b}$ J.J. Hernández-Rey, ${ }^{k}$ M. Hevinga, ${ }^{y}$ J. Hofestädt, ${ }^{g}$ C.M.F. Hugon, ${ }^{t}$ G. Illuminati, ${ }^{k}$ C.W. James, ${ }^{g}$ P. Jansweijer, ${ }^{f}$ M. Jongen, ${ }^{f}$ M. de Jong, ${ }^{f}$ M. Kadler, ${ }^{a u}$ O. Kalekin, ${ }^{g}$ A. Kappes, ${ }^{g}$ U.F. Katz, ${ }^{g}$ P. Keller, ${ }^{b}$ G. Kieft, ${ }^{f}$ D. Kießling, ${ }^{g}$ E.N. Koffeman, ${ }^{f}$ P. Kooijman, ${ }^{a q, a v}$ A. Kouchner, ${ }^{c}$ M. Kreter, ${ }^{a u}$ V. Kulikovskiy, ${ }^{b}$ R. Lahmann, ${ }^{g}$ P. Lamare, ${ }^{b}$ A. Leisos, ${ }^{j}$ E. Leonora, ${ }^{s}$ M. Lindsey Clark, ${ }^{c}$ A. Liolios, ${ }^{d}$ C.D. Llorens Alvarez, ${ }^{a m}$ D. Lo Presti, ${ }^{s}$ H. Löhner, ${ }^{y}$ A. Lonardo, ${ }^{w}$ M. Lotze, ${ }^{k}$ S. Loucatos, ${ }^{c}$ E. Maccioni, ${ }^{a j, v}$ K. Mannheim, ${ }^{a u}$ M. Manzali, ${ }^{r, n}$ A. Margiotta, ${ }^{r, a h}$ A. Margotti, ${ }^{r}$ A. Marinelli, ${ }^{a j, v}$ 
O. Mariş, ${ }^{x}$ C. Markou, ${ }^{a b}$ J.A. Martínez-Mora, ${ }^{a m}$ A. Martini, ${ }^{p}$ F. Marzaioli,,${ }^{u, a e}$ R. Mele, ${ }^{u, a i}$ K.W. Melis, ${ }^{f}$ T. Michael, ${ }^{f}$ P. Migliozzi ${ }^{u}$ E. Migneco, ${ }^{o}$ P. Mijakowski, ${ }^{a}{ }^{a}$ A. Miraglia, ${ }^{o}$

C.M. Mollo, ${ }^{u}$ M. Mongelli, ${ }^{q}$ M. Morganti, ${ }^{v, a}$ A. Moussa, ${ }^{a o}$ P. Musico, ${ }^{t}$ M. Musumeci, ${ }^{o}$ C.A. Nicolau, ${ }^{w}$ I. Olcina, ${ }^{k}$ C. Olivetto, ${ }^{c}$ A. Orlando,${ }^{o}$ A. Orzelli, ${ }^{t}$ G. Pancaldi, ${ }^{r}$ A. Paolucci, ${ }^{r}$ A. Papaikonomou, ${ }^{j}$ R. Papaleo, ${ }^{o}$ G.E. Păvălaş, ${ }^{x}$ H. Peek, ${ }^{f}$ G. Pellegrini, ${ }^{r}$ C. Pellegrino, $,{ }^{r}, a h$ C. Perrina, ${ }^{a k, w}$ M. Pfutzner, ${ }^{f}$ P. Piattelli, ${ }^{o}$ K. Pikounis, ${ }^{a b}$ G.E. Poma, ${ }^{o, a r}$ V. Popa, ${ }^{x}$ T. Pradier,${ }^{l}$ F. Pratolongo, ${ }^{t}$ G. Pühlhofer, ${ }^{e}$ S. Pulvirenti, ${ }^{o}$ L. Quinn, ${ }^{b}$ C. Racca, ${ }^{a n}$ F. Raffaelli, ${ }^{,}$ N. Randazzo, ${ }^{s}$ D. Real, ${ }^{k}$ L. Resvanis, ${ }^{a d}$ J. Reubelt, ${ }^{g}$ G. Riccobene,${ }^{o}$ C. Rossi, ${ }^{t}$ A. Rovelli, ${ }^{o}$ M. Saldaña, ${ }^{a m}$ I. Salvadori, ${ }^{b}$ D.F.E. Samtleben, ${ }^{f, z}$ A. Sánchez García, ${ }^{k}$ A. Sánchez Losa, ${ }^{q}$

M. Sanguineti, ${ }^{t}$ A. Santangelo, ${ }^{e}$ D. Santonocito, ${ }^{o}$ P. Sapienza, ${ }^{o}$ F. Schimmel,${ }^{f}$ J. Schmelling, ${ }^{f}$ J. Schnabel, ${ }^{g}$ V. Sciacca, ${ }^{o}$ M. Sedita, ${ }^{o}$ T. Seitz,${ }^{g}$ I. Sgura, ${ }^{q}$ F. Simeone, ${ }^{w}$ V. Sipala, ${ }^{s}$ B. Spisso, ${ }^{a t, u}$ M. Spurio, ${ }^{r, a h}$ G. Stavropoulos, ${ }^{a b}$ J. Steijger, ${ }^{f}$ S.M. Stellacci, ${ }^{a t}$ D. Stransky, ${ }^{g} \mathbf{M}$. Taiuti, ${ }^{t, a s}$ Y. Tayalati, ${ }^{a o, a p} \mathbf{F}$. Terrasi, ${ }^{u, a e}$ D. Tézier, ${ }^{b} \mathbf{S}$. Theraube,${ }^{b}$

P. Timmer, ${ }^{f}$ C. Tönnis, ${ }^{k}$ L. Trasatti, ${ }^{p}$ R. Travaglini, ${ }^{r}$ A. Trovato, ${ }^{o}$ A. Tsirigotis,${ }^{j}$ S. Tzamarias, ${ }^{d}$ E. Tzamariudaki, ${ }^{a b}$ B. Vallage, ${ }^{c}$ V. Van Elewyck, ${ }^{c}$ J. Vermeulen, ${ }^{f}$ F. Versari, ${ }^{r},{ }^{a h}$ P. Vicini, ${ }^{w}$ S. Viola, ${ }^{o}$ D. Vivolo, ${ }^{u, a i}$ M. Volkert, ${ }^{g}$ L. Wiggers, ${ }^{f}$ J. Wilms, ${ }^{h}$ E. de Wolf,,${ }^{f, a q}$ K. Zachariadou, ${ }^{a f}$ S. Zani, ${ }^{r, n}$ J.D. Zornoza ${ }^{k}$ and J. Zúñiga ${ }^{k}$

${ }^{a}$ Accademia Navale di Livorno, Viale Italia 72, Livorno, 57100 Italy

${ }^{b}$ Aix-Marseille Université, CNRS/IN2P3, CPPM UMR 7346, 13288, Marseille, France

${ }^{c} A P C$, Université Paris Diderot, CNRS/IN2P3, CEA/IRFU, Observatoire de Paris, Sorbonne Paris Cité, 75205 Paris, France

${ }^{d}$ Aristotle University Thessaloniki, University Campus, Thessaloniki, 54124 Greece

${ }^{e}$ Eberhard Karls Universität Tübingen, Institut für Astronomie und Astrophysik, Sand 1, Tübingen, 72076 Germany

${ }^{f}$ FOM, Nikhef, PO Box 41882, Amsterdam, 1098 DB Netherlands

${ }^{g}$ Friedrich-Alexander-Universität Erlangen-Nürnberg, Erlangen Centre for Astroparticle Physics, Erwin-Rommel-Straße 1, 91058 Erlangen, Germany

${ }^{h}$ Friedrich-Alexander-Universität Erlangen-Nürnberg, Remeis Sternwarte, Sternwartstraße 7, 96049 Bamberg, Germany

${ }^{i}$ Gran Sasso Science Institute, GSSI, Viale Francesco Crispi 7, L'Aquila, 67100 Italy

${ }^{j}$ Hellenic Open University, School of Science / Technology, Natural Sciences, Sahtouri St. / Ag. Andreou St. 16, Patra, 26222 Greece

${ }^{k}$ IFIC - Instituto de Física Corpuscular (CSIC - Universitat de València), c/Catedrático José Beltrán, 2, 46980 Paterna, Valencia, Spain

${ }^{l}$ IN2P3, IPHC, 23 rue du Loess, Strasbourg, 67037 France

${ }^{m}$ IN2P3, LPC, Campus des Cézeaux 24, avenue des Landais BP 80026, Aubière Cedex, 63171 France

${ }^{n}$ INFN, CNAF, v.le C. Berti-Pichat, 6/2, Bologna, 40127 Italy

${ }^{\circ}$ INFN, Laboratori Nazionali del Sud, Via S. Sofia 62, Catania, 95123 Italy

${ }^{p}$ INFN, LNF, Via Enrico Fermi, 40, Frascati, 00044 Italy

q INFN, Sezione di Bari, Via Amendola 173, Bari, 70126 Italy

${ }^{r}$ INFN, Sezione di Bologna, v.le C. Berti-Pichat, 6/2, Bologna, 40127 Italy

sINFN, Sezione di Catania, Via Santa Sofia 64, Catania, 95123 Italy

${ }^{t}$ INFN, Sezione di Genova, Via Dodecaneso 33, Genova, 16146 Italy 
"INFN, Sezione di Napoli, Complesso Universitario di Monte S. Angelo,

Via Cintia ed. G, Napoli, 80126 Italy

${ }^{\vee}$ INFN, Sezione di Pisa, Largo Bruno Pontecorvo 3, Pisa, 56127 Italy

${ }^{w}$ INFN, Sezione di Roma, Piazzale Aldo Moro 2, Roma, 00185 Italy

${ }^{x} I S S, 242$, Vacaresti, Bucharest, 40061 Romania

${ }^{y}$ KVI-CART University of Groningen, Groningen, The Netherlands

${ }^{z}$ Leiden University, Leiden Institute of Physics, PO Box 9504, Leiden, 2300 RA Netherlands

${ }^{a a}$ National Centre for Nuclear Research, 00-681 Warsaw, Poland

${ }^{a b}$ NCSR Demokritos, Institute of Nuclear and Particle Physics,

Ag. Paraskevi Attikis, Athens, 15310 Greece

${ }^{a c}$ NIOZ, PO Box 59, Den Burg, Texel, 1790 AB Netherlands

${ }^{a d}$ Physics Department, N. and K. University of Athens, Athens, Greece

ae Seconda Università di Napoli, Dipartimento di Matematica e Fisica, viale Lincoln 5, Caserta, 81100 Italy

af Technological Education Institute of Pireaus, Thivon and P. Ralli Str. 250, Egaleo - Athens, 12244 Greece

ag TNO, Technical Sciences, PO Box 155, Delft, 2600 AD Netherlands

${ }^{a h}$ Università di Bologna, Dipartimento di Fisica e Astronomia,

v.le C. Berti-Pichat, 6/2, Bologna, 40127 Italy

${ }^{a i}$ Università di Napoli "Federico II", Dip. Scienze Fisiche "E. Pancini",

Complesso Universitario di Monte S. Angelo, Via Cintia ed. G, Napoli, 80126 Italy

aj Università di Pisa, Dipartimento di Fisica, Largo Bruno Pontecorvo 3, Pisa, 56127 Italy

${ }^{a k}$ Università La Sapienza, Dipartimento di Fisica, Piazzale Aldo Moro 2, Roma, 00185 Italy

${ }^{a l}$ Universitat Politècnica de Catalunya, Laboratori d'Aplicacions Bioacústiques, Centre Tecnològic de Vilanova i la Geltrú, Avda. Rambla Exposició, s/n, Vilanova i la Geltrú, 08800 Spain

${ }^{a m}$ Universitat Politècnica de València, Instituto de Investigación para la Gestión Integrada de las Zonas Costeras, C/ Paranimf, 1, Gandia, 46730 Spain

${ }^{a n}$ Université de Strasbourg, Université de Haute Alsace, GRPHE,

34, Rue du Grillenbreit, Colmar, 68008 France

${ }^{a o}$ University Mohammed I, Faculty of Sciences, BV Mohammed VI, B.P. 717, R.P. 60000 Oujda, Morocco

ap University Mohammed $V$ in Rabat, Faculty of Sciences, 4 av. Ibn Battouta,

B.P. 1014, R.P. 10000 Rabat, Morocco

${ }^{a q}$ University of Amsterdam, Institute of Physics/IHEF, PO Box 94216, Amsterdam, 1090 GE Netherlands

ar University of Catania, Dipartimento di Fisica ed Astronomia di Catania, Via Santa Sofia 64, Catania, 95123 Italy

as University of Genova, Via Dodecaneso 33, Genova, 16146 Italy

${ }^{a t}$ University of Salerno and INFN Gruppo Collegato di Salerno, Department of Physics,

Via Giovanni Paolo II 132, Fisciano, 84084 Italy

au University Würzburg, Emil-Fischer-Straße 31, Würzburg, 97074 Germany

${ }^{a v}$ Utrecht University, Department of Physics and Astronomy, PO Box 80000, Utrecht, 3508 TA Netherlands

E-mail: spokesperson@km3net.de 
Abstract: The KM3NeT research infrastructure, currently under construction in the Mediterranean Sea, will host neutrino telescopes for the identification of neutrino sources in the Universe and for studies of the neutrino mass hierarchy. These telescopes will house hundreds of thousands of photomultiplier tubes that will have to be operated in a stable and reliable fashion. In this context, the stability of the dark counts has been investigated for photomultiplier tubes with negative high voltage on the photocathode and held in insulating support structures made of 3D printed nylon material. Small gaps between the rigid support structure and the photomultiplier tubes in the presence of electric fields can lead to discharges that produce dark count rates that are highly variable. A solution was found by applying the same insulating varnish as used for the high voltage bases directly to the outside of the photomultiplier tubes. This transparent conformal coating provides a convenient and inexpensive method of insulation.

KeYwords: Instrument optimisation; Large detector systems for particle and astroparticle physics; Neutrino detectors; Photon detectors for UV, visible and IR photons (gas) (gas-photocathodes, solid-photocathodes) 


\section{Contents}

1 Introduction 1

2 The measurement setup 2

3 Study of the anomalous dark count rates $\quad 3$

4 PMT insulation by using transparent conformal coating: tests and results 6

5 Conclusion $\quad 7$

\section{Introduction}

The stability of photomultiplier tubes (PMTs) is strongly influenced by electric fields. In particular, electric fields generated in the glass envelope or close surroundings of the PMT are often responsible for erratic and unstable behaviour. PMTs can be operated with a positive or a negative high voltage. The use of positive high voltage implies that the cathode is at ground potential. In this case, in order to have no field gradient and good performance it is sufficient to keep the glass case of the PMT and the material in contact with ground potential. Therefore, positive high voltage is usually the preferred mode of PMT operation [1,2]. However, for applications with the anode directly coupled to an external circuit, the use of negative high voltage is convenient. In this case, optimal and stable performance can be obtained provided that precautions are taken to eliminate electric fields in the vicinity of the PMT. Several solutions can be adopted to overcome this problem such as wrapping the PMT glass envelope with insulating tape or connecting any external conductor in contact with the PMT window or the glass envelope to cathode potential. Only good insulators should be brought into direct contact with the PMT. These precautions, although useful, have some drawbacks: wrapping insulating tape is time consuming and the connection of all surrounding materials to cathode potential implies a more complicated structure of the PMT holder. This is the challenge for the design of the KM3NeT optical modules.

KM3NeT [3] is a research infrastructure hosting the ARCA and ORCA telescopes in the Mediterranean Sea, dedicated to the identification of neutrino sources in the Universe and the study of the neutrino mass hierarchy, respectively. The working principle of the telescopes is based on the detection of Cherenkov light in the optically transparent deep-sea water induced by charged particles generated in neutrino interactions. The light is recorded by a large number of PMTs arranged in three-dimensional arrays. The basic detector element of the KM3NeT telescopes is the Digital Optical Module (DOM) [4, 5]. It comprises a support structure housing 31 three-inch PMTs within a $432 \mathrm{~mm}$ diameter glass sphere, as shown in figure 1, where the support structure is also visible. Since the hundreds of thousands of PMTs of KM3NeT will be fed with negative voltage, a support structure made of nylon has been developed to stabilise their performance. The support structure 


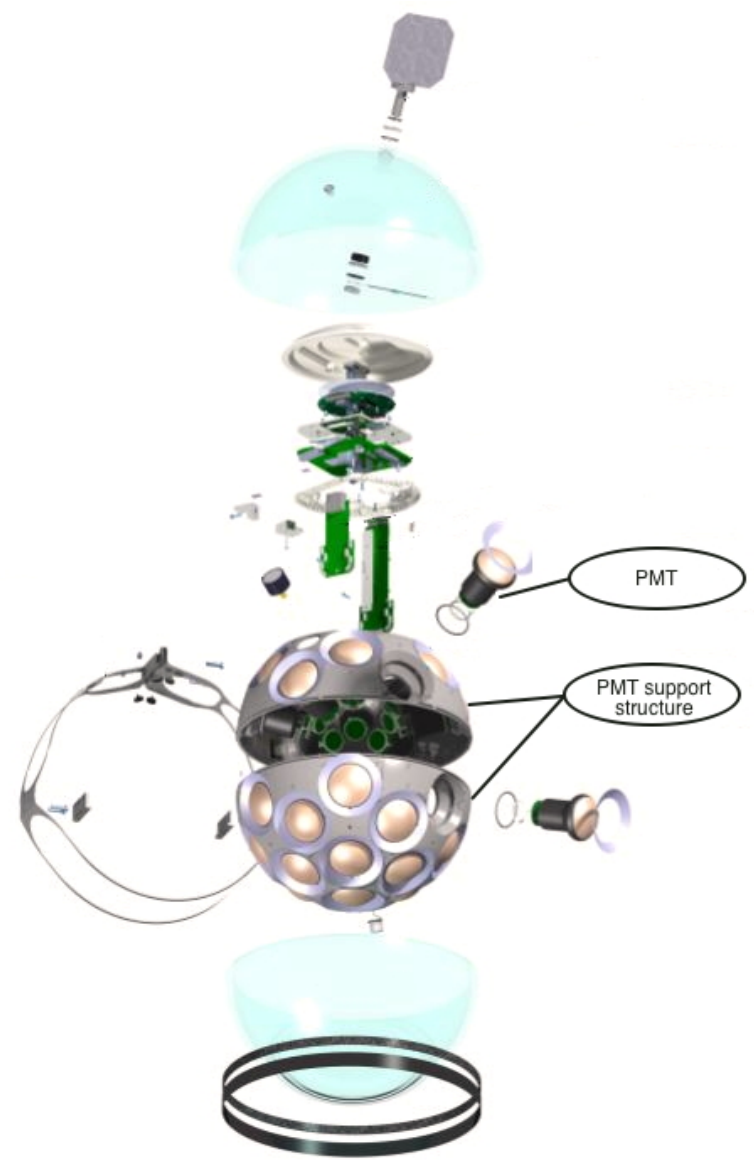

Figure 1. An exploded view of a KM3NeT DOM. A PMT and its support structure are indicated.

is manufactured by 3D printing with the selective laser sintering method. The PMT locations are tapered and a silicone O-ring tightly seals around the PMT just below its head. Even though the nylon is an excellent insulator, the dark count rates measured for PMTs (Hamamatsu R12199-02) installed in the support structure were not stable in time and well above the values measured by the vendor. Therefore, a campaign of measurements to understand the origin of the observed anomalous rates and find a remedy against it was initiated.

\section{The measurement setup}

The measurement setup for testing a PMT in its insulating holder inside a light-tight dark box [6] is shown in figure 2. A board dubbed 'MiniOctopus' provides the command signals such as the high voltage settings/thresholds and delivers the output signal to an Oscilloscope (Lecroy Wavepro SDA 760Zi). Moreover, the MiniOctopus has the connectors needed to feed $3.3 \mathrm{~V}$ to the PMT high voltage base and to the $\mathrm{I}^{2} \mathrm{C}$ bus. The NI USB- $8451 \mathrm{I}^{2} \mathrm{C}$ to USB device of National Instruments was used to connect a PC hosting the LabVIEW based software.

The dark counts as a function of time are measured using the trigger hold-off feature of the oscilloscope. 


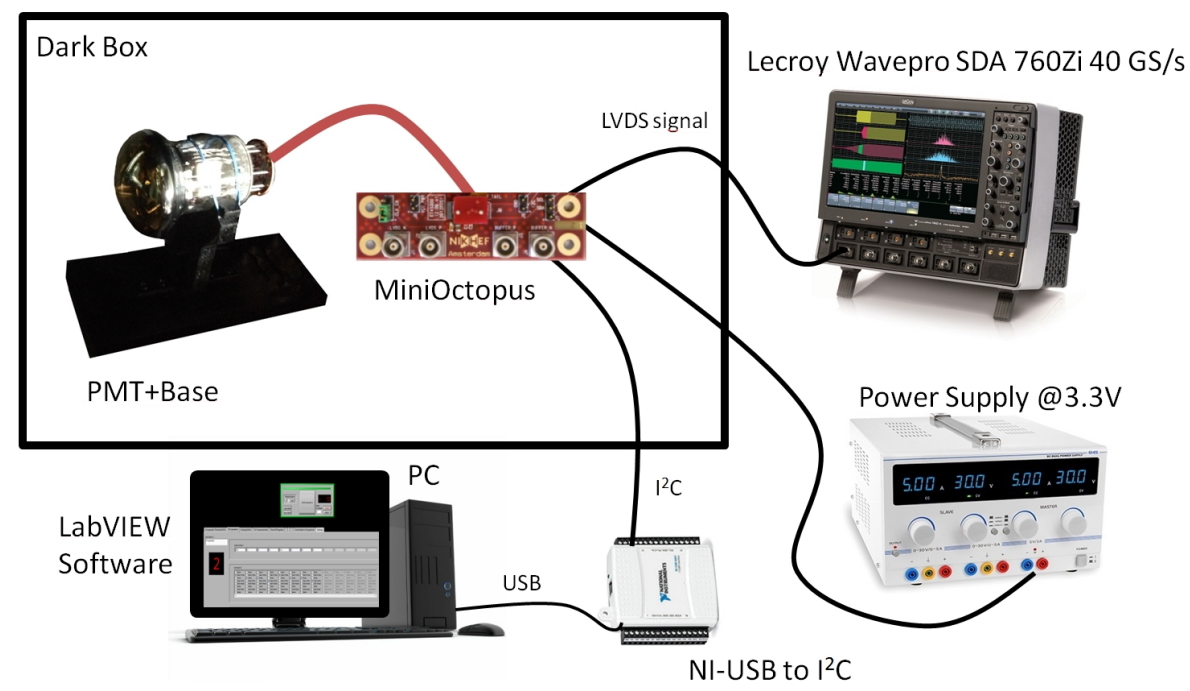

Figure 2. Setup used to measure the PMT dark count rate.
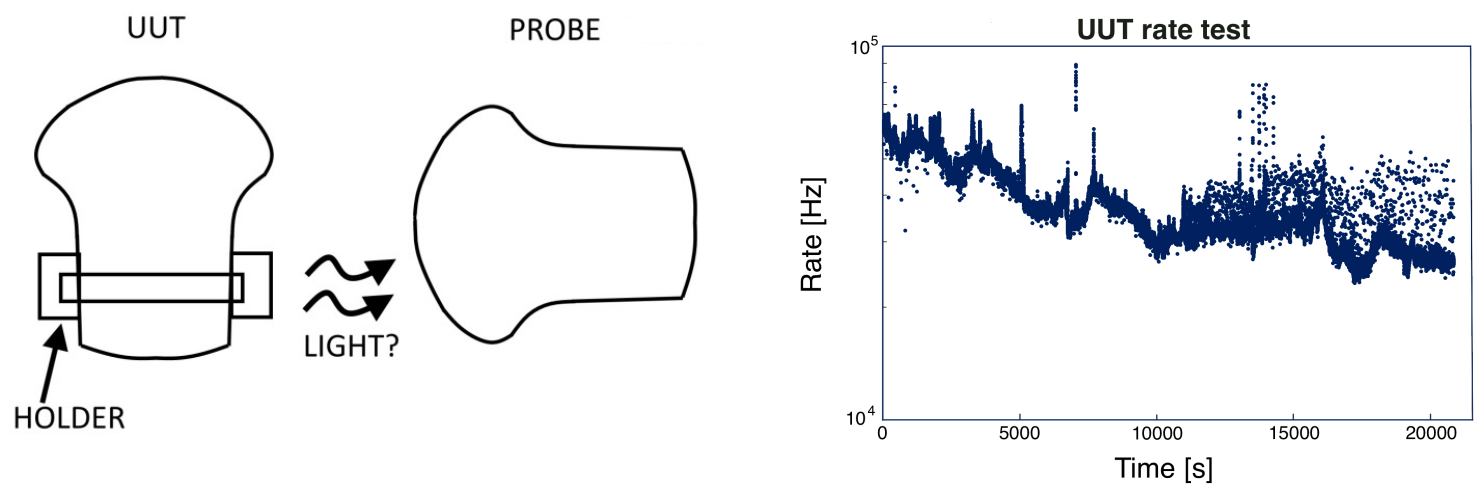

Figure 3. Left: schematic of the setup; right: dark count rate measured for a UUT supported by an insulating holder.

\section{Study of the anomalous dark count rates}

To determine the origin of the dark count anomaly, a test experiment with two PMTs arranged as shown in figure 3 was performed. Correlations between the anomalous count rates of both PMTs, which could arise either from electrostatic fields or from photons produced somewhere around the PMT, were investigated. The following nomenclature is adopted: UUT (Unit Under Test) is the PMT to be studied; PROBE is the PMT that measures the possible light produced by the UUT. In all configurations the PROBE is a PMT whose dark count rate has been measured to be within the vendor's specifications.

In the first configuration studied, the UUT was a PMT with bare glass tube supported by an insulating frame (see figure 3). The measured dark count rate of the UUT is large and unstable in time as shown in figure 3. 


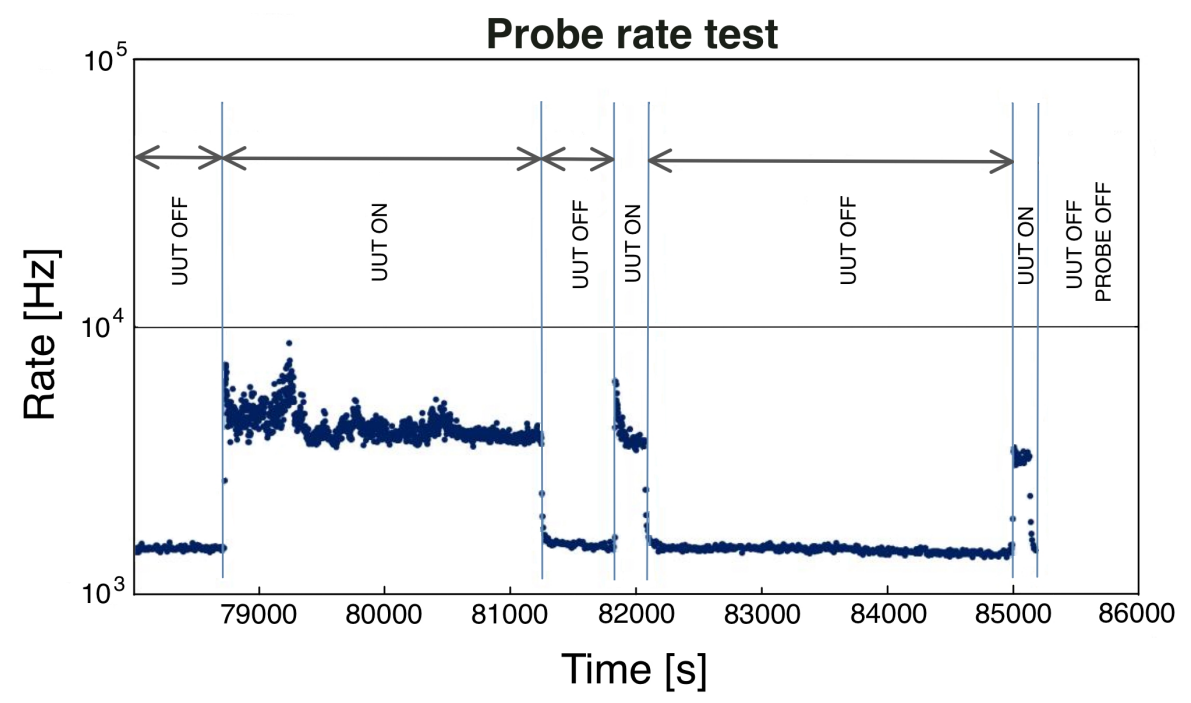

Figure 4. Count rate measured by the PROBE when the UUT is OFF and switched ON.
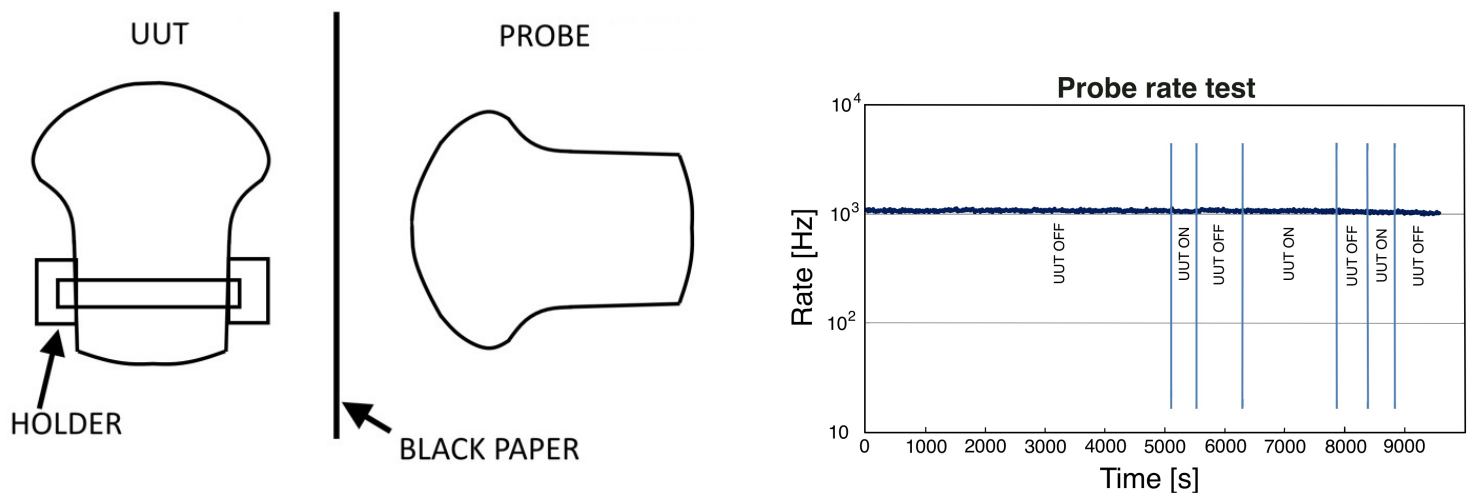

Figure 5. Left: schematic of the setup; right: count rate measured by the PROBE when the UUT is switched $\mathrm{ON}$ and OFF.

After 24 hours darkening, the count rate of the PROBE was measured iunder two configurations, the high voltage of the UUT ON and OFF (figure 4). The interesting result is that when the UUT is switched ON the rate of the PROBE increases. As soon as the UUT is switched OFF the count rate goes back to its initial value.

The correlation between the high voltage status of the UUT and the measured count rate of the PROBE could arise either from electrostatic fields created once the UUT is switched ON or from photons produced somewhere in the UUT, in particular from discharges in air due to gaps between the glass surface of the UUT and the support. As a test, a thick black paper sheet was interposed between the UUT and the PROBE and the measurement repeated. The results in figure 5 no longer show the correlation between the status of UUT and the PROBE count rate. Therefore, it was concluded that the origin of the count rate increase in the PROBE is due to photons produced in or around the UUT. 

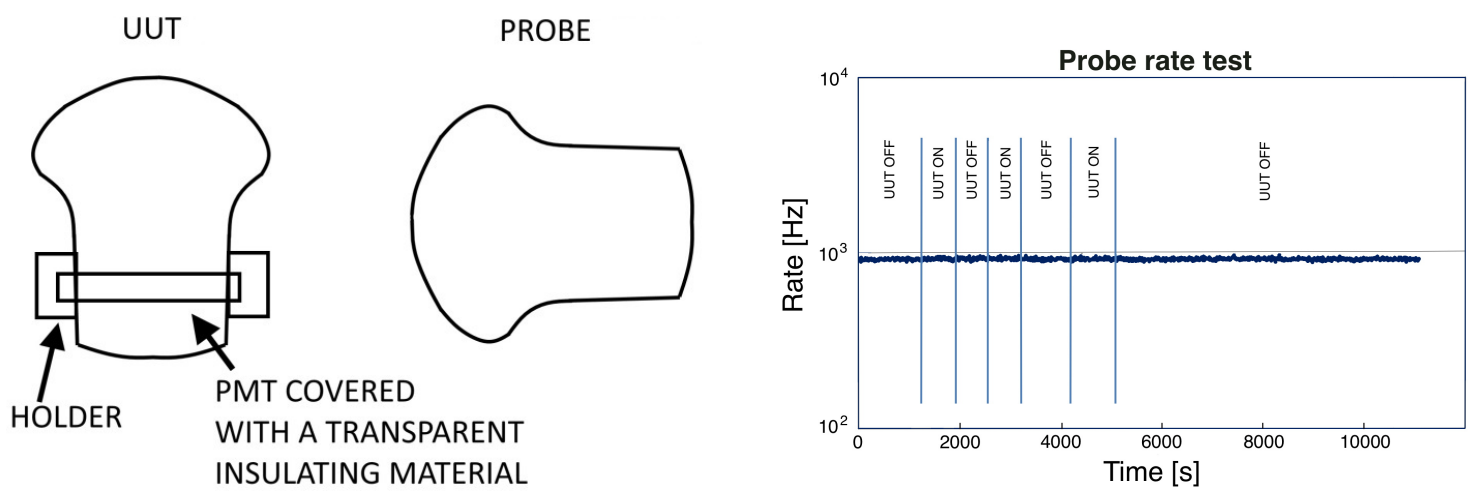

Figure 6. Left: schematic of the setup; right: count rate measured by the PROBE when the UUT is switched $\mathrm{ON}$ and OFF.
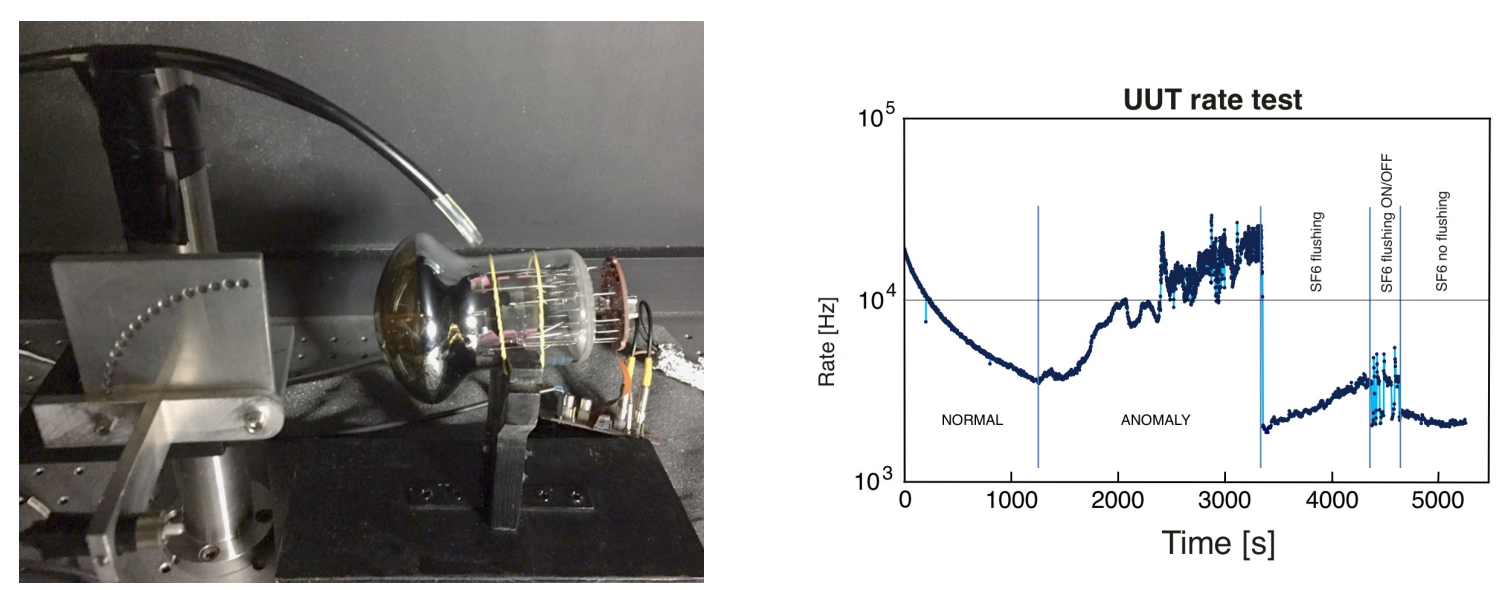

Figure 7. Left: setup to test the effect of the $\mathrm{SF}_{6}$ on the dark count rates. Note the tube on the top of the PMT used to flush the gas; right: dark count measurements before and after the $\mathrm{SF}_{6}$ flushing on the PMT.

The next step was to understand more precisely where the photons are produced. A new test with as UUT a PMT with the glass tube covered with a transparent insulating material was performed in order to detect possible photons produced by the dynodes. The setup is shown in figure 6. Over a period of 24 hours, the dark count rate of the UUT was measured at a stable value of $200 \mathrm{~Hz}$. The count rate of the PROBE shows no correlation with the high voltage status of the UUT (figure 6). Therefore, it was concluded that the same process that causes the dark count rate anomaly also produces the photons.

If the dark counts are caused by discharges in the air, the anomaly should be strongly reduced by flushing $\mathrm{SF}_{6}$, which is an electronegative gas [7], around a bare PMT. The setup to verify that and the measured dark count rate as a function of time are shown in figure 7. During the first 1000 seconds the PMT behaves normally after which the anomaly sets in and dark count rates up to 30 $\mathrm{kHz}$ were observed. As soon as the $\mathrm{SF}_{6}$ flushing is switched $\mathrm{ON}$ the dark count rate immediately reduced by about one order of magnitude. This confirms that the anomaly is due to discharges in air. 


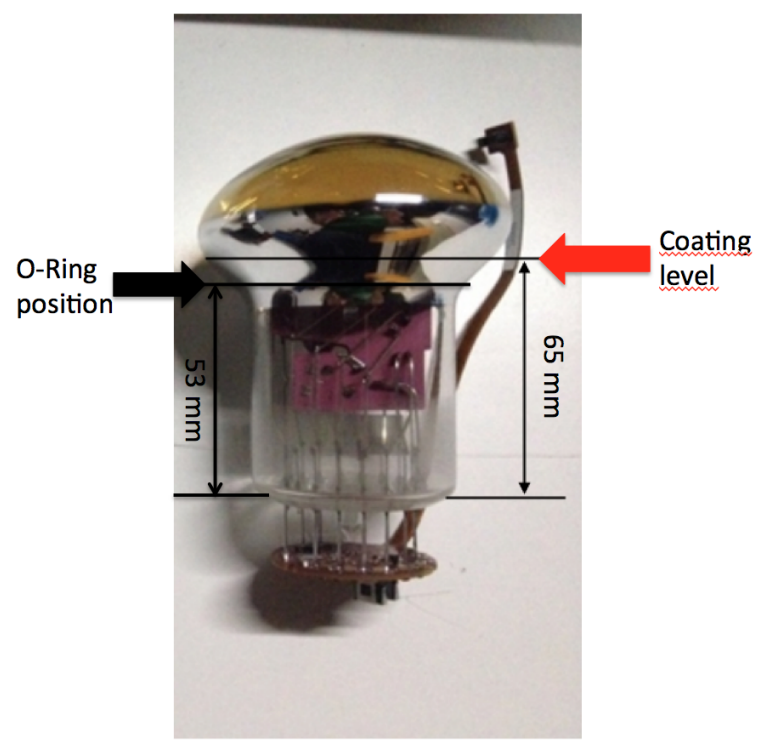

Figure 8. PMT with the indication of the level up to which the coating is applied.

\section{PMT insulation by using transparent conformal coating: tests and results}

Having understood the origin of the anomalous dark count rate, an insulating technique based on a transparent conformal coating with high surface resistivity [8] has been applied. Immersing the glass tube together with its high voltage base into the transparent conformal coating is a simple and affordable insulating method. The regions of the PMT covered with the transparent conformal coating are shown in figure 8. Note that the coating is not applied onto the photocathode area.

To determine the effectiveness of the resistive coating, the dark count rates of the same PMT were measured in the following configurations: bare, covered with insulating tape and varnished with the transparent conformal coating. The results (figure 9) show that with transparent conformal coating not only the dark counts are stable, but also the asymptotic value is reached faster than in the other configurations. The latter will shorten considerably the production time needed for the installation of hundred thousands PMTs as required for the construction of the KM3NeT telescopes.

In order to check for any heat dissipation after the application of the transparent conformal coating an infrared camera [9] was installed inside the dark box. A thermal analysis of the high voltage bases with and without coating showed that the coating affects the power dissipation only for a short time after power-up. Indeed, after 1 hour there were almost no differences between the heat map for the bases with coating or those for bases without the coating. Therefore, it was concluded that the application of the transparent conformal coating on the bases does not affect the power dissipation.

The mechanical stability of the transparent conformal coating has been verified by thermal cycling tests of the PMT in a climatic chamber [10]. The cycle from $0{ }^{\circ} \mathrm{C}$ to $50^{\circ} \mathrm{C}$ and back to $0{ }^{\circ} \mathrm{C}$ lasted about 25 minutes and was repeated 36 times for a total duration of 15 hours. After the thermal cycling the PMT coating showed no damage. This result proves that also under extreme conditions the coating does not deteriorate. 


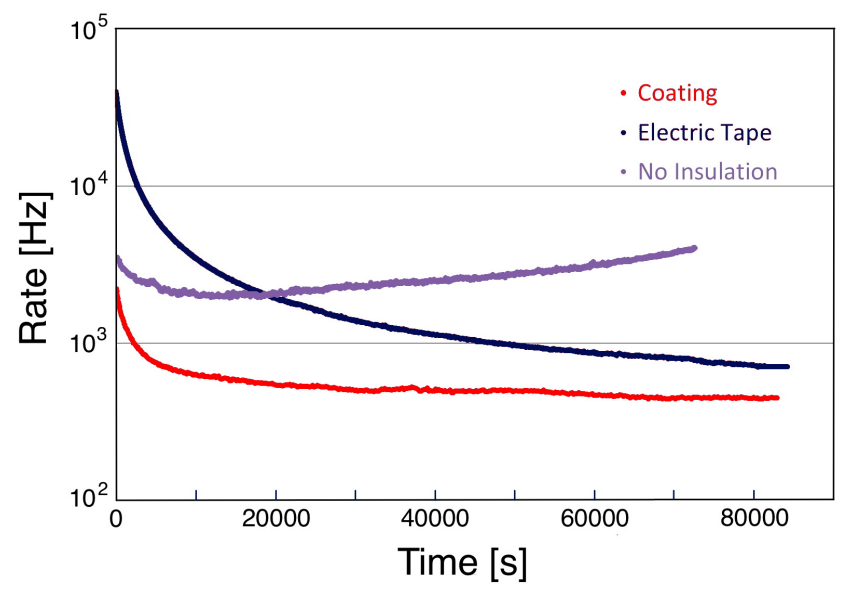

Figure 9. Dark count rate as measured for different configurations: bare PMT (blue), covered with insulating tape (black), covered with transparent conformal coating (red).

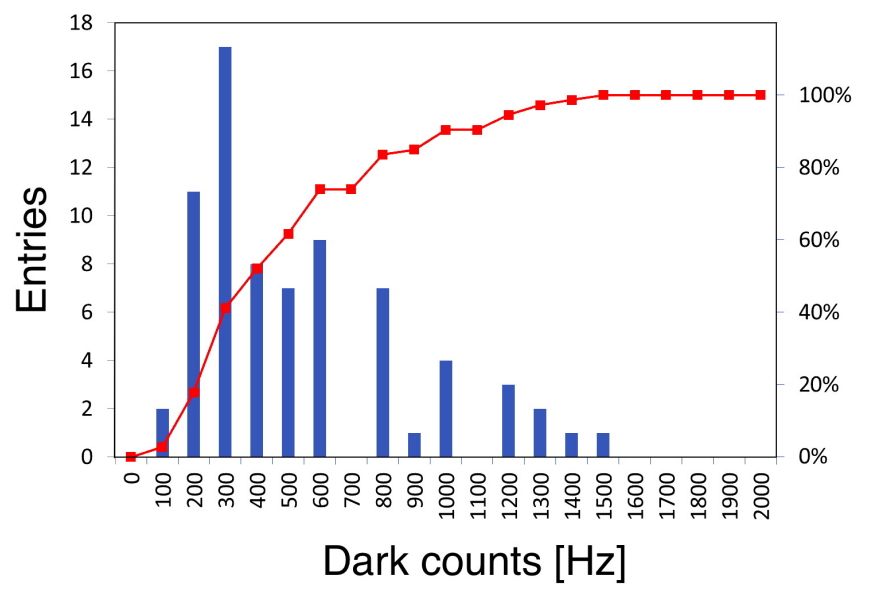

Figure 10. Measured dark count rate distribution (blue histogram). The cumulative distribution shows the fraction of PMTs below a given value.

The coating was applied to about one hundred PMTs. Dark count rates were measured and found to be well within vendor's specifications (figure 10).

\section{Conclusion}

In this paper the cause of anomalous and variable dark count rates in negatively fed PMTs for KM3NeT was investigated. A procedure to easily and effectively insulate PMTs using a highresistivity varnish has been presented. It has been shown that by using a transparent conformal coating, it is possible to stabilise the dark count rate without affecting the other PMT parameters.

Currently, in order to avoid anomalous behaviour in PMTs fed with negative voltage one must either rely on solutions from manufacturing companies with external circuits that complicate the use of instrumentation or cover the PMT envelope with insulating tape. The insulating method presented in this paper has several advantages with respect to the presently adopted solutions: 
- Better insulation. To ensure a good stability of the dark counts it is essential that the insulating material is in close contact with the PMT. This is achieved best with transparent conformal coating.

- Ease of implementation. Since the same varnish is used to insulate the high voltage base, the PMT and base can be coated during the same operation. This allows for reduction of integration time and cost reduction compared to the use of insulating tape.

- Uniformity of thickness of the insulating material. The application of the coating only increases the PMT thickness by a few tenths of a millimetre and ensures an excellent uniformity of thickness.

\section{Acknowledgments}

The authors acknowledge the financial support of the funding agencies: Centre National de la Recherche Scientifique (CNRS), Commission Européenne (FEDER fund and Marie Curie Program), Institut Universitaire de France (IUF), IdEx program and UnivEarthS Labex program at Sorbonne Paris Cité (ANR-10-LABX-0023 and ANR-11-IDEX-0005-02), France; The General Secretariat of Research and Technology (GSRT), Greece; Istituto Nazionale di Fisica Nucleare (INFN), Ministero dell'Istruzione, dell'Università e della Ricerca (MIUR), Italy; Agence de l'Oriental and CNRST, Morocco; Stichting voor Fundamenteel Onderzoek der Materie (FOM), Nederlandse organisatie voor Wetenschappelijk Onderzoek (NWO), the Netherlands; National Authority for Scientific Research (ANCS), Romania; Plan Estatal de Investigación (refs. FPA2015-65150-C3-1-P, -2-P and -3-P, (MINECO/FEDER)), Severo Ochoa Centre of Excellence and MultiDark Consolider (MINECO), and Prometeo and Grisolía programs (Generalitat Valenciana), Spain.

\section{References}

[1] Hamamatsu Photonics Photomultiplier Tubes Handbook, https://www.hamamatsu.com.

[2] ET Enterprises Photomultiplier Tubes Handbook, http://www.et-enterprises.com.

[3] KM3Net collaboration, S. Adrián-Martínez et al., Letter of intent for KM3NeT 2.0, J. Phys. G 43 (2016) 084001 [arXiv: 1601.07459$].$

[4] KM3NeT collaboration, S. Adrián-Martínez et al., The prototype detection unit of the KM3NeT detector, Eur. Phys. J. C 76 (2016) 54 [arXiv: 1510.01561$].$

[5] KM3NET collaboration, S. Adrián-Martínez et al., Deep sea tests of a prototype of the KM3NeT digital optical module, Eur. Phys. J. C 74 (2014) 3056 [arXiv: 1405.0839].

[6] C.M. Mollo et al., A new instrument for high statistics measurement of photomultiplier characteristics, 2016 JINST 11 T08002 [arXiv: 1604 .03922].

[7] W.M. Haynes ed., CRC Handbook of Chemistry and Physics, 97th edition, CRC Press, 24 June 2016, pg. 2652 [ISBN: 9781498754286].

[8] Coating Electronics varnish \& Lacquer, http://docs-europe.electrocomponents.com/webdocs/0d80/0900766b80d8031c.pdf.

[9] http://www.flir.com.

[10] http://www.angelantonitestechnologies.com. 